\title{
Early Diagnosis of Cardiac Toxicity Related to Antineoplastic Treatment
}

\author{
Maria Maiello ${ }^{1}$, Rakesh K. Sharma ${ }^{2}$, Marco Matteo Ciccone ${ }^{3}$, Humananth K. Reddy ${ }^{4}$, \\ Pasquale Palmiero ${ }^{5^{*}}$ \\ ${ }^{1}$ Cardiology Department, ASL BR, Brindisi, Italy; ${ }^{2}$ Cardiology Chair, University of Arkansas for Medical Sciences, El Dorado, USA; \\ ${ }^{3}$ Cardiology Chair, University of Bari, Bari, Italy; ${ }^{4}$ Clinical Professor of Medicine, University of St. Louis, Associate Medical Director \\ Medical Center of South Arkansas St. Louis, Arkansas, USA; ${ }^{5}$ Contract Professor of Cardiology, University of Heart Station, \\ Brindisi, Italy. \\ Email: pasquale.palmiero@yahoo.it
}

Received April 13 $3^{\text {th }}, 2011$; revised May 23 $3^{\text {rd }}, 2011$; accepted May 31 ${ }^{\text {st }}, 2011$.

\begin{abstract}
Background: breast cancer because of radiotherapy and/or chemotherapy causes cardiac disease, often it occurs on women just affected by hypertension and/or diabetes. All these conditions may affect left ventricular (LV) geometry, mass and diastolic function. The purpose of this study is to early detect these affections to improve heart failure prevention. Patients and methods: 134 women, affected by breast cancer, underwent to conventional transthoracic echocardiography (TTE) and pulse wave tissue Doppler imaging (PW-TDI). A control group (CG) of 80 women unaffected by breast cancer, hypertension and diabetes was enrolled. Results: 54 of 134 women (40.2\%), were affected by hypertension or diabetes too (Prone Group $=P G), 8$ by both, $80(59.8 \%)$ were free $(F G)$. Mean age of all patients was 45.4 years. The rates of $L V$ eccentric hypertrophy and $L V$ distolic dysfunction were statistically significant higher on $F G$ group than controls, and on PG group than FG group. Conclusions: an abnormal LV diastolic function is more common among women affected by breast cancer after treatment than in general population, the same for LV eccentric hypertrophy but at a lower rate. $40 \%$ of women were affected by hypertension, diabetes or both, and as expected they have a higher rate of $L V$ eccentric hypertrophy and diastolic dysfunction. This high prevalence of $L V$ eccentric hypertrophy and diastolic dysfunction, on asymptomatic women, affected by breast cancer, is a predictor of heart failure; Doppler-echo techniques may be helpful in early diagnosis.
\end{abstract}

Keywords: Breast Cancer, Radiotherapy, Chemotherapy, Hypertension, Diabetes, Left Ventricular Geometry, Left Ventricular Mass, Diastolic Function, Eccentric Hypertrophy

\section{Introduction}

Cancer treatment actually is a combination of surgery associated to chemotherapy [1] and radiotherapy [2] to prolong life duration and quality. Unfortunately this combination can cause cardiotoxicity, it can precociously on set but also arise after many years from therapy interruption [3] Many different chemotherapeutic agents can determine cardiovascular complications such as heart failure for left ventricular function impairment, all kinds of coronary artery diseases and, at a lower rate, thromboembolism, and arrhythmias [4]. The same happens for radiotherapy because of mediastinal irradiation [5]. It is important to early recognize cardiotoxicity onset because of its significant impact on the cardiac function and survival of cancer patients. To assess LV function, a base- line evaluation of LV ejection fraction (EF) is always obtained, before chemo and/or radio therapy start, and it is recommended that the same methodology be used for serial assessment [6] It was first established in clinical practice since 1979 [7], based on their experiences, algorithms have been developed for serial monitoring of LVEF during chemiotherapy [8,9]. Measuring systolic function through evaluation of the LVEF with echocardiography is one of the most commonly used measurements in monitoring and diagnosing chemotherapy-induced cardiomyopathy; however, it is not sensitive for early detection of pre-clinical cardiac disease (subclinical), and it is influenced by contractility and pre-load /afterload effects leading to transient changes. Therefore, other measurements of LV function (e.g., E/A ratio) have been used to detect early cardiotoxicity in addition to 
LVEF [10]. Due to an increasing aging population of patients with cancer and the introduction of many new cancer therapies, breast cancer often occurs on women just affected by hypertension and/or diabetes [11] All these conditions may affect left ventricular (LV) geometry, mass and diastolic function, but in what proportion it is unknown. Early detection of these conditions may contribute to improve heart failure prevention. The purpose of our study was to determine the prevalence of left ventricular changes of geometry, mass and diastolic dysfunction on a population of women affected by breast cancer, submitted to radiotherapy and chemotherapy, who present preserved LVEF.

\section{Patients and Methods}

We screened by our heart station 134 consecutive women, all submitted to radiotherapy and/or chemotherapy, because of breast cancer, with a mean age of $52 \pm 7$ years, affected by breast cancer and a group of 80 consecutive women unaffected by breast cancer, hypertension and diabetes, mentioned as control group, population's characteristics are reported on Table 1. During last three years they underwent to conventional transthoracic echocardiography (TTE), it was performed by GE Vivid 3 Expert echocardiography, an echo-Doppler system equipped with a multifrequency transducer. Exclusion criteria were diagnosis of: LV ejection fraction $<50 \%$, wall motion abnormalities, severe valvular disease, atrial fibrillation and pulmonary hypertension, estimated from the tricuspid regurgitation velocity by modified Bernoulli equation. All patients were examined in a partially supine position, with the head of the examining table elevated by $30^{\circ}$. Recording followed a standardized protocol, the parasternal acoustic window was used to record at least 10 consecutive beats, in two-dimensional and M-mode recordings, to measure the $\mathrm{LV}$ internal diameter and wall thicknesses, at or just below the tips of the anterior mitral valve leaflets in both the long- and short-axis views. The apical acoustic window was used to record at least 10

Table 1. Characteristics of enrolled women.

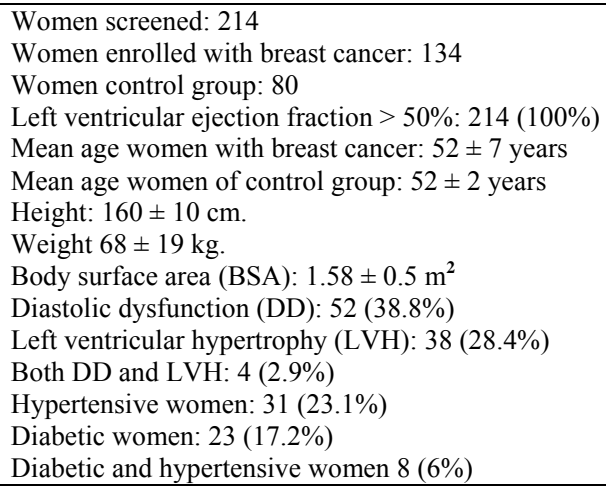

cycles of two- and four-chamber images to assess LV wall motion. Pulsed Doppler sample volumes were placed at the center of the mitral annulus in the fourchamber view to record diastolic transmitral blood flow. The Doppler beam was aligned to produce the narrowest possible angle between the beam and the blood flow vector. Pulsed-wave (PW) Doppler was performed in the apical 4-chamber view to obtain mitral inflow velocities to assess LV filling [12]. Continuous Wave (CW) Doppler to assess peak E (early diastolic) and A (late diastolic) velocities. On mitral inflow we measured peak early filling (E-wave) and late diastolic filling (A-wave) velocities, E/A ratio, deceleration time (DT) of early filling velocity, and isovolumic relaxation time (IVRT) [13]. PW DTI is performed in apical views to acquire mitral annular velocities, sample volume was positioned at or 1 $\mathrm{cm}$ within the septal and lateral insertion sites of the mitral leaflets. To measure systolic and early $\left(E^{\prime}\right)$ and late (A') diastolic velocities. For the assessment of global LV diastolic function, we acquired and measured tissue Doppler signals at the septal and lateral sides of the mitral annulus and their average. $\mathrm{E}^{\prime}$ was used to correct for the effect of LV relaxation on mitral E velocity, and the $E / E^{\prime}$ ratio was applied for the prediction of LV filling pressures. On all patients $E / E^{\prime}$ ratio was calculated. An average ratio $\leq 8$ identified patients with normal LV filling pressures, whereas a ratio $\geq 13$ indicates an increase in LV filling pressures. 86 When the ratio is between 9 and 13, a change in E/A ratio with the Valsalva maneuver of $\geq 0.5$ and maximal LA volume $\geq 34 \mathrm{~mL} / \mathrm{m}^{2}$ were indicative of increased LV filling pressures other measurements are essential. The grading scheme was mild or grade I (impaired relaxation pattern: mitral E/A ratio, < 0.8 , DT $>200 \mathrm{~ms}$, IVRT $\geq 100 \mathrm{~ms}$, annular $\mathrm{E}^{\prime}<8 \mathrm{~cm} / \mathrm{s}$, $\mathrm{E} / \mathrm{E}^{\prime}$ ratio $\left.<8\right)$, moderate or grade II (PNF: mitral E/A ratio $0.8-1.5, \mathrm{E} / \mathrm{E}^{\prime}$ ratio 9 to 12 , and $\mathrm{E}<8 \mathrm{~cm} / \mathrm{s}$ ), and severe (restrictive filling) or grade III (E/A ratio $\geq 2$, DT $<160 \mathrm{~ms}$, IVRT $\leq 60 \mathrm{~ms}$, systolic filling fraction $\leq 40 \%$, $\mathrm{E} / \mathrm{E}^{\prime}$ ratio $>13$ [14]. Adequate measurements of diastolic function were obtained in all patients. Hypertension and or diabetic status was assessed after at least 6 months of antihypertensive or antidiabetic therapy. LV mass was estimated by M-mode echocardiography. Patients with segmental LV dysfunction were excluded. LVMI was derived by dividing LV mass by body surface area. LVH was defined as LV mass index $>110 \mathrm{~g} / \mathrm{m}^{2}$ for women and $>134 \mathrm{~g} / \mathrm{m}^{2}$ for men [12,15-17]. LVEF was determined on M-mode and checked on B-mode by Simpson's rule. Left ventricular geometry was assessed on the basis of relative wall thickness, classed as concentric when $\geq 0.45$ and as eccentric when $\leq 0.34$ [18].

To determine intra- and inter-observer variability, each of two investigators duplicated each measurement in 10 
randomly chosen patients to ensure that a correlation coefficient of 0.9 or more was obtained by linear regression analysis. Measurements were made with a computerized review station equipped with digitizing tablet and monitor screen overlay. LV internal dimension and wall thicknesses were measured at end diastole and end systole according to the American Society of Echocardiography recommendations $[19,20]$. When optimal orientation of imaging views could not be obtained, as is common in subjects who are overweight, correctly oriented linear dimension measurements were made with two-dimensional imaging by the leading-edge technique recognized by the American Society of Echocardiography $[21,22]$. Doppler transaortic flow was performed in the view in which peak flow velocity was maximal by tracing the black-white interface of the Doppler flow envelope after calibration for velocity and time [23,24].

\subsection{Calculation of Derived Variables}

End-diastolic LV dimensions were used to calculate LV mass by an anatomically validated formula [12,15-17]. LV relative wall thickness (RWT) was calculated by formula as: left ventricle RWT $=$ IVS + PW/LVEDD, where IVS is interventricular septum thickness, $\mathrm{PW}$ is posterior wall thickness and LVEDD is LV end diastolic diameter. f left ventricular RWT was greater than 0.45 ; it was classified as concentric geometry of LV and if less than 0.34 ; classified as eccentric geometry [25].

\subsection{Statistical Analysis}

The relationships between concentric lv hypertrophy (lvh) patients and eccentric lvh patients for all the above-mentioned echo findings were assessed by the chi-square test for non-parametric analysis and student's t-test for parametric analysis. A $p$ value $<0.05$ was considered significant. Multivariate analysis was performed.

\section{Results}

All patients were enrolled during a period of 3 years, 54 of 134 women $(40.2 \%)$, were affected by hypertension and/or diabetes too, 8 of them by both, these women had been on anti-hypertensive and or anti-diabetic medication for almost 6 months prior to enrolment. No relation has been found between medications used and outcome, possibly because of a high frequency of combination therapy or serial monotherapy with different drugs.. The mean age of all patients was 52.7 years, 53.7 on patients without diabetes or hypertension, mentioned as free group (FG), 51.6 on women with almost one of two conditions, prone group (PG) and 52.2 on control group (CG). We diagnosed LV hypertrophy on 10 women of FG (12.5\%), 24 of PG (44.4\%), $p<0.02$ and 2 of CG (2.5\%), $p<0.007$ vs FG and $p<0.01$ vs CG. According to LV geometry,
LV eccentric hypertrophy was present on 8 women of FG $(10 \%), 18$ of PG $(33,4 \%), p<0.01$ and 2 of CG $(2.5 \%), p$ $<0.006$ vs FG and $p<0.01$ vs CG; LV concentric hypertrophy was present on 2 women of FG $(2.5 \%), 6$ of PG $(11.1 \%), p<0.05$ and none of CG; LV concentric remodeling, (i.e. concentric geometry without LVH), was present on 1 women of FG (1.2\%), 3 of PG $(5.5 \%), p<$ 0.003 and none of CG, Figure 1. LV diastolic dysfunction as filling impairment was on 20 women of FG $(25 \%)$, on 30 of PG (55.5\%), p $<0.04$ and on 2 of CG (3.7\%), p $<0.04$ vs $\mathrm{PG}$ and $p<0.05$ vs FG, diastolic dysfunction was considered mild in all case and in no case LV filling pressure were increased. 1 woman of FG and 3 of PG were affected by $\mathrm{LVCH}$ and LV diastolic dysfunction) $\mathrm{DD}$, but this number of patients is too little to be reliable for any evaluation. Coronary artery disease, without regional wall motion abnormalities was diagnosed on 2 patients affected by diabetes and hypertension. The women of all 3 groups were not statistically different in terms of age and prevalence of smoking.

\section{Discussion}

The main outcome of our study is that patients treated by chemotherapy and radiotherapy, present many different changes of LV mass, geometry and function, in absence of other risk factors for these changes. LV mass is increased, LV geometry became often eccentric and sometimes concentric, LV filling is impaired also if they are asymptomatic and despite a preserved LV ejection fraction. For all these modification there is a statistical significant different rate between our patients and general population. The most frequent pattern is LV diastolic dysfunction, followed by LV eccentric hypertrophy, at a lower rate. This suggests that on these patients cancer treatment may early impair LV diastolic filling and/or may cause LV dilation, determining LV eccentric hypertrophy, while they are asymptomatic for cardiac disease.

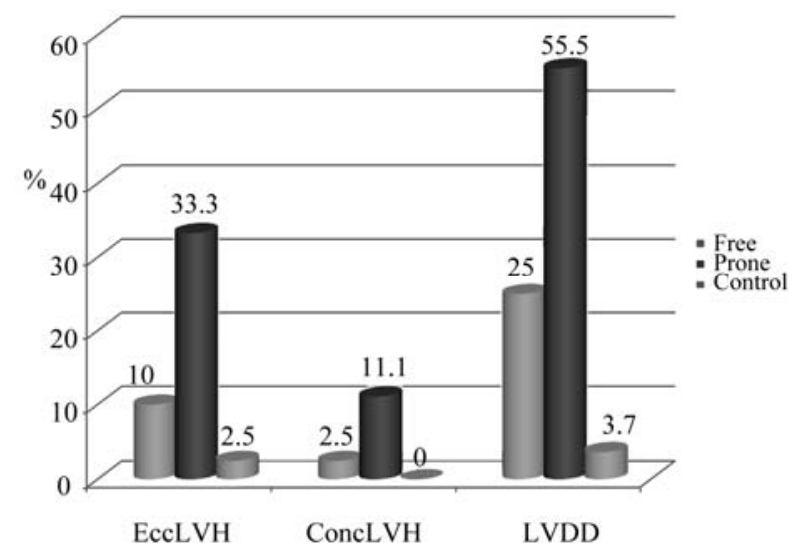

Figure 1. Prevalence of left ventricular eccentric or concentric hypertrophy and diastolic dysfunction on our population. 
LV filling pressure was normal in all patients, but we have to consider that we are looking for early cardiac dysfunction. We know that a combined systo-diastolics dysfunction is relatively common during on women who underwent to cancer therapy [26-28], so it is reasonable to perform serial evaluation of LVEF [6], as prescribed by current guidelines, but when LVEF became impaired, it occurs a rapid development of severe heart failure. Early diagnosis of LV diastolic dysfunction and/or LV eccentric hypertrophy can be achieved by a simple tool such M-B mode echocardiogram, associated to PW and TDI Doppler, and it is more sensitive than LVEF in the early detection of LV unfavourable changes in geometry, as assessed by LV relative wall thickness and function. LV geometry eccentric pattern is common among our patients, not the same for concentric pattern, so we can suppose that cancer therapy doesn't determine changes on peripheral resistances, but directly affect myocardial cells, the same explanation can be accounted for LV diastolic dysfunction not due to increased peripheral resistances but to direct myocardial cell damage. The biological mechanisms underlying cancer therapy-associated cardiac dysfunction remain to be fully elucidated, but generation of reactive oxygen species (ROS) and induction of cardiac myocyte apoptosis are hypothesized to play a central role. Concerning, chemotherapy ROS may continue to be produced by a drug retained within myocytes contributing to late-occurring cardiovascular injury [29]. Also about radiotherapy, the generation of ROS is thought to play a major role [30]. Another important outcome is that all above mentioned changes in LV mass, geometry and function are present at a statistical significant different higher rate among a population of women treated by chemotherapy and radiotherapy, but just affected by cardiovascular risk factors as diabetes and or hypertension [31], compared with our population of women treated for cancer but free from diabetes and or hypertension. So that the cardiac unfavorable changes, due to cancer therapy, add up to those due to diabetes and or hypertension, increasing their cardiovascular risk of events [31,32]. All these patients need of a careful monitoring of heart condition to prevent heart failure and other cardiac events. The main limitation of our study is the limited number of patients enrolled, so we cannot individuate subgroups according to different treatments, not even we can differentiate diabetic from hypertensive women, otherwise it is one of the first studies concerning breast cancer patient treated by both chemotherapy and radiotherapy, with preserved LV ejection fraction.

\section{Conclusions}

An abnormal diastolic function is more common among women affected by breast cancer and treated for it than in general population, the same happens for LV eccentric hypertrophy but at a lower rate. $40 \%$ of women were affected by hypertension, diabetes or both, and they have a higher rate of LV eccentric hypertrophy and diastolic dysfunction than women treated for cancer but unaffected. We know that patients affected by these LV changes had worse event-free survival than patients with normal LV mass, geometry and function. LV eccentric hypertrophy and diastolic dysfunction are predictors of heart failure on asympomatic women. Screening with Doppler echo techniques may be helpful in identifying patients at high risk for subsequent cardiac events.

\section{Disclosures}

All authors have no financial disclosures, they report no biomedical financial interests or potential conflicts of interest.

\section{Acknowledgements}

The authors thank the nurses Grazia Quaranta, Emmia Donativo and Anna Paola Gennaro, for the support to the survey of the clinical data.

\section{REFERENCES}

[1] M. N. Levine and T. Whelan, "Adjuvant Chemotherapy for Breast Cancer-30 Years Later," The New England Journal of Medicine, Vol. 355, No. 18, 2006, pp. 19201922. doi:10.1056/NEJMe068204

[2] Early Breast Cancer Trialists' Collaborative Group, "Effects of Radiotherapy and Surgery in Early Breast Cancer. An Overview of the Randomized Trials," The New England Journal of Medicine, Vol. 333, No. 22, 1995, pp. 1444-1455. doi:10.1056/NEJM199511303332202

[3] M. J. Hooning, et al., "Long-Term Risk of Cardiovascular Disease in 10-year Survivors of Breast Cancer," Journal of the National Cancer Institute, Vol. 99, No. 5, 2007, pp. 365-375. doi:10.1093/jnci/djk064

[4] T. Hampton, "Cancer Therapy Can Be Hard on the Heart: Researchers Aim to Explain-and Avoid-Cardiotoxicity," Journal of the American Medical Association, Vol. 303, No. 11, 2010, pp. 1019-1020. doi:10.1001/jama.2010.269

[5] P. A. Heidenreich, et al., "Diastolic Dysfunction after Mediastinal Irradiation," American Heart Journal, Vol. 150, No. 5, 2005, pp. 977-982. doi:10.1016/j.ahj.2004.12.026

[6] M. T. Meinardi, et al., "Evaluation of Long Term Cardiotoxicity after Epirubicin Containing Adjuvant Chemotherapy and Locoregional Radiotherapy for Breast Cancer Using Various Detection Techniques," Heart, Vol. 88 , No. 1, 2002, pp. 81-82. doi:10.1136/heart.88.1.81

[7] J. Alexander, et al., "Serial Assessment of Doxorubicin Cardiotoxicity with Quantitative Radionuclide Angiocardiography," The New England Journal of Medicine, Vol. 
300 , No. 6, 1979, pp. 278-283. doi:10.1056/NEJM197902083000603

[8] R. G. Schwartz, et al., "Congestive Heart Failure and Left Ventricular Dysfunction Complicating Doxorubicin Therapy. Seven-Year Experience Using Serial Radionuclide Angiocardiography," American Journal of Medicine, Vol. 82, No. 6, 1987, pp. 1109-1118. doi:10.1016/0002-9343(87)90212-9

[9] L. Mosca, et al., "Evidence-Based Guidelines for Cardiovascular Disease Prevention in Women: 2007 Update," Journal of the American College of Cardiology, Vol. 49, No. 11, 2007, pp. 1230-1250. doi:10.1016/j.jacc.2007.02.020

[10] W. I. Ganz, et al., "Review of Tests for Monitoring Doxorubicin-Induced Cardiomyopathy," Oncology, Vol. 53, No. 6, 1996, pp. 461-470. doi:10.1159/000227621

[11] R. Yancik, et al., "Effect of Age and Comorbidity in Postmenopausal Breast Cancer Patients Aged 55 Years and Older," Journal of the American Medical Association, Vol. 285, No. 7, 2001, pp. 885-892. doi:10.1001/jama.285.7.885

[12] C. P. Appleton, et al., "Doppler Evaluation of Left and Right Ventricular Diastolic Function: A Technical Guide for Obtaining Optimal Flow Velocity Recordings," Journal of the American Society of Echocardiography, Vol. 10, No. 3, 1997, pp. 271-292. doi:10.1016/S0894-7317(97)70063-4

[13] J. W. Ha, et al., "Triphasic Mitral Inflow Velocity with Middiastolic Filling: Clinical Implications and Associated Echocardiographic Findings," Journal of the American Society of Echocardiography, Vol. 17, No. 5, 2004, pp. 428-431. doi:10.1016/j.echo.2004.02.007

[14] S. F. Nagueh, et al., "Recommendations for the Evaluation of Left Ventricular Diastolic Function by Echocardiography," Journal of the American Society of Echocardiography, Vol. 22, No. 2, 2009, pp. 107-133. doi:10.1016/i.echo.2008.11.023

[15] R. B. Devereux and N. Reichek, "Echocardiographic Determination of Left Ventricular Mass in Man. Anatomic Validation of the Method," Circulation, Vol. 55, No. 4, 1977, pp. 613-618.

[16] H. W. Hense, et al., "The Associations of Body Size and Body Composition with Left Ventricular Mass: Impacts for Indexation in Adults," Journal of the American College of Cardiology, Vol. 32, No. 2, 1998, pp. 451-457. doi:10.1016/S0735-1097(98)00240-X

[17] G. P. Aurigemma, M. R. Zile and W. H. Gaasch, "Contractile Behavior of the Left Ventricle in Diastolic Heart Failure: With Emphasis on Regional Systolic Function," Circulation, Vol. 113, No. 2, 2006, pp. 296-304. doi:10.1161/CIRCULATIONAHA.104.481465

[18] G. de Simone, et al., "Evaluation of Concentric Left Ventricular Geometry in Humans: Evidence for Age-Related Systematic Underestimation," Hypertension, Vol. 45, No. 1, 2005, pp. 64-68.

[19] D. J. Sahn, et al., "Recommendations Regarding Quantitation in M-mode Echocardiography: Results of a Survey of Echocardiographic Measurements," Circulation, Vol. 58, No. 6, 1978, pp. 1072-1083.

[20] M. M. Redfield, et al., "Burden of Systolic and Diastolic Ventricular Dysfunction in the Community: Appreciating the Scope of the Heart Failure Epidemic," Journal of the American Society of Echocardiography, Vol. 289, No. 2, 2003, pp. 194-202. doi:10.1001/jama.289.2.194

[21] N. B. Schiller, et al., "Recommendations for Quantitation of the Left Ventricle by Two-Dimensional Echocardiography. American Society of Echocardiography Committee on Standards, Subcommittee on Quantitation of Two-Dimensional Echocardiograms," Journal of the American Society of Echocardiography, Vol. 2, No. 5, 1989, pp. 358-367.

[22] R. A. Nishimura, and A. J. Tajik, "Evaluation of Diastolic Filling of Left Ventricle in Health and Disease: Doppler Echocardiography is the Clinician's Rosetta Stone," Journal of the American College of Cardiology, Vol. 30, No. 1, 1997, pp. 8-18.

[23] J. Dubin, et al., "Comparative Accuracy of Doppler Echocardiographic Methods for Clinical Stroke Volume Determination," American Heart Journal, Vol. 120, No. 1, 1990, pp. 116-123. doi:10.1016/0002-8703(90)90168-W

[24] S. R. Ommen, et al., "Clinical Utility of Doppler Echocardiography and Tissue Doppler Imaging in the Estimation of Left Ventricular Filling Pressures: A Comparative Simultaneous Doppler-Catheterization Study," Circulation, Vol. 102, No. 15, 2000, pp. 1788-1794.

[25] A. Ganau, et al., "Patterns of Left Ventricular Hypertrophy and Geometric Remodeling in Essential Hypertension," Journal of the American College of Cardiology, Vol. 19, No. 7, 1992, pp. 1550-1558. doi:10.1016/0735-1097(92)90617-V

[26] J. J. Doyle, et al., "Chemotherapy and Cardiotoxicity in Older Breast Cancer Patients: A Population-Based Study," Journal of Clinical Oncology, Vol. 23, No. 34, 2005, pp. 8597-8605. doi:10.1200/JCO.2005.02.5841

[27] E. A. Perez, et al., "Effect of Doxorubicin Plus Cyclophosphamide on Left Ventricular Ejection Fraction in Patients with Breast Cancer in the North Central Cancer Treatment Group N9831 Intergroup Adjuvant Trial," Journal of Clinical Oncology, Vol. 22, No. 18, 2004, pp. 3700-3704. doi:10.1200/JCO.2004.03.516

[28] E. T. Yeh, "Cardiotoxicity Induced by Chemotherapy and Antibody Therapy," Annual Review of Medicine, Vol. 57, 2006, pp. 485-498. doi:10.1146/annurev.med.57.121304.131240

[29] D. Lebrecht, et al., "Time-Dependent and Tissue-Specific Accumulation of mtDNA and Respiratory Chain Defects in Chronic Doxorubicin Cardiomyopathy," Circulation, Vol. 108, No. 19, 2003, pp. 2423-2429. doi:10.1161/01.CIR.0000093196.59829.DF

[30] M. S. Anscher and Z. Vujaskovic, "Mechanisms and Potential Targets for Prevention and Treatment of Normal Tissue Injury after Radiation Therapy," Seminars in Oncology, Vol. 32, No. 2 (Supplement), 2005, pp. S86-91. doi:10.1053/i.seminoncol.2005.03.015 
[31] D. M. Lloyd-Jones, et al., "Prediction of Lifetime Risk for Cardiovascular Disease by Risk Factor Burden at 50 Years of Age," Circulation, Vol. 113, No. 6, 2006, pp. 791-798. doi:10.1161/CIRCULATIONAHA.105.548206

[32] P. M. Ridker, et al., "Development and Validation of
Improved Algorithms for the Assessment of Global Cardiovascular Risk in Women: The Reynolds Risk Score," Journal of the American Society of Echocardiography, Vol. 297, No. 6, 2007, pp. 611-619. doi:10.1001/jama.297.6.611 